\title{
Correction to: Review of surgical techniques and guide for decision making in the treatment of benign parotid tumors
}

\author{
Georgios Psychogios ${ }^{1,11}$ (1) C Christopher Bohr ${ }^{2}$. Jannis Constantinidis ${ }^{3} \cdot$ Martin Canis $^{4} \cdot$ Vincent Vander Poorten $^{5,11}$. \\ Jan Plzak $^{6} \cdot$ Andreas Knopf $^{7} \cdot$ Christian Betz $^{8} \cdot$ Orlando Guntinas-Lichius ${ }^{9,11}$. Johannes Zenk ${ }^{10,11}$
}

Published online: 29 August 2020

○) Springer-Verlag GmbH Germany, part of Springer Nature 2020

\section{Correction to: European Archives of Oto-Rhino-Laryngology https://doi.org/10.1007/s00405-020-06250-x}

In the original publication of the article, one of the author's name was published incorrectly as "Christopher Betz". The correct name is Christian Betz.

In addition, the affiliation of the authors Christian Betz and Andreas Knopf were published incorrectly. The correct affiliation of Christian Betz is "Department of Otorhinolaryngology, Universitätsklinikum Hamburg-Eppendorf, Martinistr. 52, 20246 Hamburg, Germany" and the affiliation of Andreas Knopf is "Department of Otorhinolaryngology, Head and Neck Surgery, Faculty of Medicine, University of Freiburg, Freiburg, Germany".

These corrections were updated in the original article.

The original article can be found online at https://doi.org/10.1007/ s00405-020-06250-x.

Georgios Psychogios

gpsychogios@uoi.gr

1 Department of Otorhinolaryngology and Head and Neck Surgery, University Hospital Ioannina, Leof. Stavrou Niarchou, 45500 Ioannina, Greece

2 Department of Otorhinolaryngology and Head and Neck Surgery, University Hospital Regensburg, Franz-Josef-Strauss-Allee 11, 93053 Regensburg, Germany

3 1st Department of ORL, Head and Neck Surgery, Aristotle University, AHEPA Hospital, Thessaloniki, Greece

4 Department of Otorhinolaryngology and Head and Neck Surgery, LMU Klinikum, Marchioninistr. 15, 81377 Munich, Germany

5 Otorhinolaryngology-Head and Neck Surgery and Department of Oncology, Section Head and Neck Oncology, University Hospitals Leuven, KU Leuven, Louvain, Belgium
Finally, the caption of Fig. 1 was published incorrectly. The correct caption is "Capsular dissection in patient with pleomorphic adenoma of the deep lobe of the right PG undergoing TP".

Publisher's Note Springer Nature remains neutral with regard to jurisdictional claims in published maps and institutional affiliations.

6 Department of Otorhinolaryngology and Head and Neck Surgery, 1st Faculty of Medicine, Charles University, Motol University Hospital, V Uvalu 84, 15006 Prague 5, Czech Republic

7 Department of Otorhinolaryngology, Head and Neck Surgery, Faculty of Medicine, University of Freiburg, Freiburg, Germany

8 Department of Otorhinolaryngology, Universitätsklinikum Hamburg-Eppendorf, Martinistr. 52, 20246 Hamburg, Germany

9 Department of Otorhinolaryngology-Head and Neck, Surgery, Jena University Hospital, Jena, Germany

10 Department of Otorhinolaryngology and Head and Neck Surgery, University Hospital Augsburg, Sauerbruchstraße 6, 86179 Augsburg, Germany

11 Multidisciplinary Salivary Gland Society (http://www.msgs.international), Geneva, Switzerland 